This technique has been used on several nebulae to produce temperature maps, using the [OIII] line ratios, and density maps, using the [SII] lines. (Paper to be submitted to Mon.Not.R.Astr.Soc.)

\title{
DISCUSSION
}

Osterbrock: Can you say quantitatively what is the discrimination of the $\lambda 6713$ filter against $\lambda 6716$ for instance, and of the $\lambda 4363$ against $\mathrm{HgI} \lambda 4358$ (if it is a problem) and $\mathrm{H} \gamma$ ?

Worswick: The bandpasses of the $6713 \AA$ and $6717 \AA$ filters, which are double half wave interference filters, drop to the order of $1 \%$ of the peak transmission at about $7 \AA$ from the line. Contamination from the mercury emission line was fairly low at the Lowell Observatory site. Aller: It would be extremely valuable to compare the electron temperature derived from [NII] $\lambda 5755$ vs. 6584 , and [OIII] $\lambda 4363$ vs. 5007 on a point to point basis within the nebular images. We could then ascertain the scale of the temperature fluctuations from point to point.

Worswick: The project is feasible providing you have the correct filter, enough observing time and computer time.

Terzian: The maps you showed can now be compared with the radio synthesis maps and the projected distribution of the interstellar extinction can be derived.

Dopita: At Mt. Stromlo I have undertaken a program of absolute surface brightness measurements of planetaries using the SEC Vidicon photometer developed there along with the 74" Multi-Channel Scanner. These give contour maps of surface brightness directly with single frame signal to noise ratio better than 30 . I have observed approximately 30 planetary nebulae in $\mathrm{H} \beta$ and [OIII].

THE ELECTRON TEMPERATURE IN THE $\mathrm{He}^{++}$REGIONS IN PLANETARY NEBULAE

\author{
R. Tylenda \\ Nicolaus Copernicus University \\ Torun, Poland
}

A method of determination of the electron temperature in the $\mathrm{He}^{++}$ regions in planetary nebulae from observed intensities of [NeV] $\lambda 3426$ and [NeIV] $\lambda 4714-25$ relative to HeII $\lambda 4686$ is presented. It has been used for 22 planetary nebulae for which the observational data have been taken from Kaler's Catalogue (Ap. J. Supp1., 31, 517). The elec-

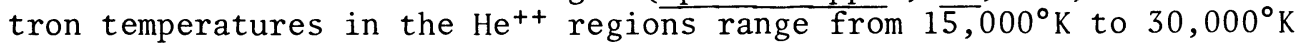


for most of the studied planetaries and are usually significantly higher than the temperatures determined from [OIII] lines which refer to the $\mathrm{He}^{+}$regions. A correlation between the electron temperatures and effective temperatures of central stars has been found; the planetaries with hotter nuclei have slightly higher electron temperatures in the $\mathrm{He}^{++}$ regions. Finally, intensities of ultraviolet lines of [NeV] $\lambda 2975$ and [NeIV] $\lambda 2424$ have been predicted for the studied nebulae.

\section{SPECTROPHOTOMETRY OF SIX PLANETARY NEBULAE}

C.T. Hua

Laboratoire d'Astronomie Spatiale, Marseille, France

In addition to numerous emission lines arising from the ionization of chemical elements, well-exposed nebular spectrograms reveal a Balmer continuum in emission hitherto attributed to the second level radiative recombination of the hydrogen atoms and to the two-photon contribution. The very intense lines with regard to the adjacent continuous spectrum have been easily measured by many workers for identification, Balmer decrements, line ratios ... whereas few data are available involving continuum measurements. As well as the mean slope of the continuum, the Balmer discontinuity is very sensitive to the physical conditions prevailing and the presence of dust in the nebulae.

We present recent spectrophotometric data obtained at the Haute Provence Observatory using the new method of comparison between an extended source (nebula) and star. This study is made possible by means of the Chalonge spectrograph, the plate holder of which widens by oscillating motions of the stellar spectrum in the red. The spectrophotometric procedures are similar to those described previously for the Orion Nebula. The results are given in terms of absolute gradients defined within the spectral range $\lambda \lambda 5000-3100$ and the strength of the Balmer jump. The electron temperature derived from the continuum parameters is compared with values obtained by other authors. 